\title{
JOÃO PEREIRA DA SILVA, FRANCISCO ADOLFO DE VARNHAGEN ET LES MALHEURS DE L'HISTOIRE MODERNE DU BRÉSIL
}

\author{
Armelle Enders \\ Universidade Paris IV-Sorbonne
}

\section{Résumé}

Peu d'historiens se sont consacrés à l'histoire du Brésil indépendant sous l'Empire et João Manuel Pereira da Silva et Francisco Adolfo de Varnhagen constituent deux exceptions à cette règle. Véritable succès de librairie, le livre de Pereira da Silva História da Fundação do Império Brasileiro, en 7 gros volumes publiés à partir de 1864, est cependant vite discrédité, ainsi que son auteur, qui tombe dans l'oubli. Varnhagen ne doit pas sa gloire à ses travaux sur l’indépendance, édités longtemps après sa mort. Ces mésaventures historiographiques posent le problème de la légitimité de l'histoire contemporaine et des difficiles rapports de celle-ci avec la politique et la monarchie brésilienne.

\section{Resumo}

Poucos historiadores se dedicaram à história do Brasil independente sob o Império; João Manuel Pereira da Silva e Francisco Adolfo de Varnhagen constituem duas exceções a esta regra. Verdadeiro sucesso de vendas, o livro de Pereira da Silva, História da Fundação do Império Brasileiro, em sete grossos volumes, publicados a partir de 1864, foi rapidamente desprezado, assim como o seu autor, que caiu no esquecimento. Quanto a Varnhagen, ele não deve sua glória aos textos que escreveu sobre o a Independência, editados muito tempo após a sua morte.

Essas desventuras historiográficas colocam o problema da legitimidade da história contemporânea e das relações difíceis desta com a política e a monarquia brasileira. 
En 1847, un concours académique organisé par l'Instituto Histórico e Geográfico Brasileiro trancha la querelle des Anciens et des Modernes à propos de la manière dont on devait écrire l'histoire du Brésil. Deux érudits avaient envoyé leur dissertation sur ce thème emprunté à Lucien de Samosate et présenté chacun une méthode pour rendre compte de "a história antiga e moderna do Brasil, organizada com tal sistema que nela se compreendam as suas partes política, civil, eclesiástica e literária”․ L’IHGB écarta la proposition de Barros e Couto, qui invitait à suivre le schéma des Décadas de Tite-Live, comme incapable d'accoucher d'une “história como genero filósofico, como se deve exigir atualmente" ${ }^{2}$, et il prima celle du naturaliste bavarois Karl von Martius qui faisait du croisement des trois "races" qui peuplaient le Brésil le fil conducteur tant du récit historique que de l'accomplissement du pays. Martius connaissait aussi le texte de Lucien et, comme ce dernier, recommandait à l'historien d'utiliser un langage clair, simple et noble à la fois, de manière à être compris de tous. Pour le reste, le savant bavarois se détournait des principes antiques et retenait ceux formulés par ses contemporains ; il prônait plus l'amour du trône et de la religion que celui de la seule vérité ; il s’inspirait, assez banalement, de la théorie de l'histoire fondée sur l'opposition entre les “races conquérantes” et les “races conquises” qui avait servi, dans l'Europe romantique, à expliquer la genèse des peuples modernes, de Walter Scott à Augustin Thierry.

L'IHGB se félicita d'avoir suscité une dissertation aussi lumineuse qu'ambitieuse et l'adopta comme son programme officiel. L'histoire conservait la portée morale que lui prêtaient les Anciens, la "maîtresse de vie” cicéronienne, le bréviaire d'exemples héroïques puisés dans les vies des ancêtres, mais la portée patriotique l'emportait sur les autres dimensions. L'histoire devait inventer ce peuple moderne en voie de formation, le peuple brésilien, et assurer la cohésion du jeune, immense et fragile Empire du Brésil en répandant l’amour de la Patrie, confondue avec la monarchie.

Il y eut cependant un écart considérable entre les objectifs que se fixa l'IHGB et la concrétisation de ceux-ci. Si la revue de l'IHGB — de loin la plus durable et la plus stable du Brésil impérial — rassemble des milliers d'articles et contributions diverses qui témoignent de la richesse de la production historique,

\footnotetext{
${ }^{1}$ 168a sessão do IHGB, 10 de junho de 1847, Revista do $I H G B, n^{\circ} 6,1847$, p. 279.

${ }^{2}$ Ibidem. Grifo no original.
} 
peu d'œuvres ont finalement émergé et sont parvenues jusqu'au XXI ${ }^{\mathrm{e}}$ siècle en faisant encore figure de référence.

Parmi ces auteurs, Francisco Adolfo de Varnhagen (1816-1878), qui affecta de négliger les leçons de Martius, s’est imposé comme le “père de l'histoire” au Brésil et l'un des “constructeurs" ou "interprètes” de la nation, essentiellement grâce aux deux volumes de l'História Geral do Brasil, publiés à Madrid en 1854 et 1857, qui portent sur la période coloniale, mais aussi à de très nombreux ouvrages comme celui consacré à la guerre contre les Hollandais ${ }^{3}$. Un de ses successeurs, João Capistrano de Abreu (1853-1927) continue à jouir de l'estime de la profession pour avoir élargi le questionnaire historique à la société et à la culture matérielle quand Varnhagen s'en tenait à l'État royal. Capistrano de Abreu a laissé de très nombreuses et percutantes notes de lecture et des études importantes comme Capitulos de história colonial (1907) ou le posthume Caminhos antigo e povoamento do Brasil (1930), mais l'œuvre historique ellemême laisse un fort goût d'inachevé. Entre Varnhagen et Capistrano, se glisse peut-être Joaquim Norberto da Sousa Silva (1820-1891), une figure secondaire dans les ouvrages historiographiques, mais néanmoins auteur d'articles fort honorables, notamment sur Tiradentes. Les autres illustrations de l'IHGB sont passés à la postérité, non pour leurs travaux historiques, mais pour leur œuvre littéraire (Gonçalves de Magalhães, Joaquim Manuel de Macedo) ou leur rôle dans les Beaux-Arts (Manoel de Araújo Porto Alegre).

La plupart des livres marquants rédigés sous D. Pedro II portent sur la période coloniale, avant donc, la naissance effective de l’État-nation brésilien. L’indépendance, moment fondateur pour tous les nouveaux Etats d'Amérique du Sud, semble négligée. La situation est d'autant plus paradoxale que l’IHGB fut précisément créé par des hommes comme Januário da Cunha Barbosa qui jouèrent un rôle très important dans la rupture du Brésil et du Portugal et la fondation de l'Empire.

Cette lacune rend encore plus mystérieux l'oubli presque complet dans lequel est tombé João Manuel Pereira da Silva (1817-1897), pourtant l'un des plus prolixes historiens du XIX ${ }^{\mathrm{e}}$ siècle brésilien et pratiquement le seul à consacrer ses efforts, non à la période coloniale, mais au Brésil indépendant.

\footnotetext{
${ }^{3}$ VARNHAGEN, Francisco Adolfo de. História das lutas com os holandeses no Brasil, desde 1624 a 1654, Viena (Áustria), 1871.
} 
En 1864 sort en effet le premier des sept tomes totalisant plus de deux mille cinq pages que comptera l'História da Fundação do Império Brasileiro. Les tomes 2, 3, 4 et 6 paraissent en 1865. Le septième achève la série en 1868. La première édition, tirée par B.L. Garnier à trois mille exemplaires ${ }^{4}$, est tellement couronnée de succès qu'elle s’épuise et nécessite une nouvelle édition “revista, correta e acrescentada" en trois gros volumes en 1870-1871, puis en 1877. Ainsi, l'História da fundação do Império Brasileiro est incontestablement l'un des principaux best-sellers du règne de dom Pedro II, ce que même les nombreux détracteurs de Pereira da Silva ne manquent pas de souligner.

João Manuel Pereira da Silva, membre de l'IHGB depuis 1838, continua sur sa lancée et compléta l'étude des années 1808-1825 par un livre de près de cinq cents pages sur le Segundo Período do Reinado de D. Pedro I no Brasil (1825-1831), publié en 1871, et un autre tout aussi volumineux sur l'histoire des régences, História do Brasil de 1831 à 1840 (1879). Francisco Adolfo de Varnhagen, sans doute piqué au vif par le travail de son collègue, s’attela lui aussi à un livre sur l’indépendance, dont il annonçait la publication imminente en 1876, mais qui ne parut que bien après sa mort. La revue de l'IHGB utilisa en effet cet inédit, l'História da Independência do Brasil, até o reconhecimento pela antiga metrópole, comprehendo, separadamente, a dos successos occorridos em algumas províncias até essa data, annotée par le baron do Rio Branco, pour célébrer le centenaire de la naissance de Varnhagen en $1916^{5}$. Cet ouvrage n’est pas le plus apprécié de Varnhagen et contribua peu à sa gloire, demeurée intacte jusqu'à aujourd'hui.

Il y a lieu de croire que l'histoire moderne - au sens d'histoire contemporaine, voire d'histoire du temps présent - reste sous D. Pedro II une histoire maudite, qui apporta un succès triomphal à João Pereira da Silva auprès du public, mais lui valut également des volées de bois vert de la part de ses confrères et le priva de postérité. La damnatio memorice dont Pereira da Silva fut victime reflète tant l'ambiance impitoyablement compétitive du milieu intellectuel sous l'Empire, que le caractère explosif que pouvait revêtir l'histoire du règne de D. Pedro $\mathrm{I}^{\mathrm{er}}$ sous celui de son successeur.

\footnotetext{
${ }^{4}$ BLAKE, Augusto Victorino Alves Sacramento. Dicionário bibliográfico brasileiro. Rio de Janeiro: Tipografia Nacional, vol.3, p. 479 et sq.,1883-1902 (réédition fac-similé 1970).

${ }^{5}$ RIHGB, t.133, 1916, p.7-596.
} 


\section{Le vicomte et le conseiller d'État}

Varnhagen (né en 1816) et Pereira da Silva (né en 1817) appartiennent exactement à la même génération, laquelle suit celle de l'indépendance et accède aux affaires au moment de la Majorité anticipée de dom Pedro II. Ils partagent, en outre, des origines étrangères.

Le futur vicomte de Porto Seguro, fils d'un ingénieur venu de Hesse et passé au service de la monarchie portugaise et d'une mère portugaise, fut naturalisé et eut à cœur, tout au long, de sa vie, de multiplier les preuves de son ardeur patriotique. Dans les innombrables disputes qu'engagea l'historien, dont le caractère épouvantable était notoire, ses origines étaient parfois stigmatisées. Ainsi, le général Abreu e Lima que Varnhagen avait accusé de plagiat dans les pages de la revue de l'IHGB en 1845, traita son confrère de pédant et d'ignorant et le qualifia de "fils d'Allemand et d'une dame qui n'était pas Brésilienne"6.

João Manuel Pereira da Silva descendait pour sa part d'un négociant portugais de la place de Rio de Janeiro et d'une mère portugaise mais née au Brésil. Sa nationalité ne posa pas de problème, même si une grande partie de son œuvre montre beaucoup d'attention et de sympathie sur la situation politique et sociale du Portugal et qu'il fut récompensé par le gouvernement portugais pour avoir dénoué quelques litiges entre les deux pays.

La formation des deux hommes diffère. Varnhagen passa quelques années au Real Colégio Militar de Lisbonne et rentra au Brésil où il entra dans le corps impérial des ingénieurs avant de verser dans la diplomatie et de passer la plus grande partie de sa vie hors du Brésil. Il mourut à Vienne en juin 1878, ministre plénipotentiaire auprès de la Cour des Habsbourg.

Pereira da Silva alla étudier le droit à Paris, obtint son diplôme en 1838, revint à Rio de Janeiro et se consacra jusqu'en 1850 à la carrière d'avocat, où il semble qu'il amassa une belle fortune, et à la vie politique fluminense et brésilienne au sein du parti conservateur. Il fut longtemps député, tant à l'assemblée de la province de Rio de Janeiro qu’à l'assemblée générale de l’Empire, devint conseiller d’État, acheva sa carrière comme sénateur, mais ne fut jamais ministre.

Contrairement à Varnhagen, qui aspirait à recevoir un titre de noblesse et fut finalement créé baron, puis vicomte de Porto Seguro, Pereira da Silva ne

\footnotetext{
${ }^{6}$ Rapporté par Pedro Lessa lors de la session commémorative de l’IHGB pour le centenaire de la naissance de Varnhagen, 17 février 1916, RIHGB, t.80, vol. 134, 1916, p. 622.
} 
fut pas anobli. Lui-même se montrait très critique dans ses écrits à l'égard de l'introduction d'une noblesse titrée au Brésil et considérait que cette institution, dépassée et au mieux inutile, était en outre étrangère aux traditions du pays.

Pereira da Silva collectionna en revanche les appartenances à toutes sortes de sociétés savantes et littéraires dans le vieux comme dans le nouveau monde: Academia Real das Ciências de Lisbonne, Société de géographie et des économistes de Paris, Arcádia de Roma, Academia Real de História (Madrid), Institut historique de Paris... et fut l'un des fondateurs de l'Académie brésilienne des lettres, premier titulaire du fauteuil n ${ }^{\circ} 34$ ayant pour patron Sousa Caldas. Il avait participé aussi, dans sa jeunesse, à l'émergence du romantisme brésilien en écrivant dans l'éphémère revue littéraire Niterói, placée sous le patronage de Manuel de Araújo Porto Alegre et Gonçalves de Magalhães, ses collègues de l’IHGB.

Varnhagen et Pereira da Silva obtinrent l'un comme l'autre la reconnaissance académique, mais suscitèrent aussi la controverse. L'História Geral do Brasil fut fraîchement accueillie et la revue de l'IHGB n'en rendit même pas compte $^{7}$. Les reproches faits à Varnhagen poursuivront longtemps celui-ci. La qualité de son style était controversée, son dédain affiché pour le programme défini par Karl von Martius et son hostilité militante contre les indigènes du Brésil en plein mouvement indianiste lui valurent des inimitiés persistantes ${ }^{8}$, mais la postérité effaça ces réserves et couronna le vicomte de Porto Seguro dès sa mort en 1878.

\section{Qu'il est périlleux d'éfudier l'histoire de son temps}

Il en alla tout autrement pour João Manuel Pereira da Silva. En 1847, les membres de l'Instituto apprécièrent la collection de biographies de personnages de l'époque coloniale que Pereira da Silva avait intitulé O Plutarco Brasileiro, mais Inocêncio Francisco da Silva dressa la liste des “erreurs” contenues dans l'ouvrage et on jugea le titre "pompeux". Pereira da Silva rectifia donc les "erreurs" et adopta pour la nouvelle édition le titre tout aussi pompeux de Os varões ilustres do Brasil.

\footnotetext{
${ }^{7}$ CEZAR, Temístocles Américo Correa. L'écriture de l'histoire au Brésil au XIX $X^{e}$ siècle. Essai sur une rhétorique de la nationalité. Le cas Varnhagen. Paris, Thèse de doctorat, École des Hautes Études en Sciences Sociales, 2002.

${ }^{8}$ Pedro Lessa, session commémorative de l'IHGB pour le centenaire de la naissance de Varnhagen, 17 février 1916, RIHGB, t.80, vol. 134, 1916, p. 630 et sq.
} 
Le premier volume de l'História da fundação do Império brasileiro en 1864 reçut à son tour un accueil plutôt flatteur du Premier Secrétaire de l'IHGB, le chanoine Fernandes Pinheiro, qui rendit ainsi hommage dans un style fleuri à son confrère : "Nas delicadas mãos do nosso esclarecido colega adquire cada documento duplicado valor ; semelhante á concha fossil, na qual descobre o paleontólogo os vestígios do molusco que o habitára em épocas ante-históricas (...). Honra ao nosso distinto patrício, que tão bem compreendeu as leis da história, e que, trilhando a vereda dos Niebuhrs, Mommsen, Guizot, Thiers, Macaulay e Herculano, novos e sazonados frutos nos promete do seu robusto

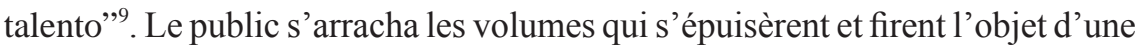
nouvelle édition, phénomène exceptionnel pour une œuvre aussi copieuse.

João Manuel Pereira da Silva passa pourtant rapidement du Capitole à la roche Tarpéienne. Apparemment, ce fut la publication en 1871 de son livre sur la seconde période du règne de Dom Pedro $\mathrm{I}^{\mathrm{er}}$ au Brésil ${ }^{10}$ qui déclencha la fureur et anéantit sa réputation comme historien. Commencèrent les réclamations individuelles des personnages qui s’estimaient mal lotis. Ainsi, le 30 septembre 1872, Conrad Jacob Niemeier, présenté à plusieurs reprises par Pereira da Silva comme un partisan de l'absolutisme, déposa à l'IHGB quelques feuillets intitulés Impugnação da obra do Exm. Sr. Conselheiro João Manuel Pereira da Silva, segundo período do reinado de D. Pedro I no Brasil, narrativa histórica, 1871, na parte relativa ao comandante de armas e presidente da comissão militar da província de Ceará, de 1824 a 1828.

Quelques années plus tard, c'est la qualité même d'historien qui lui fut contestée par ses honorables collègues. À l'occasion d'une réédition de l'História da fundação do Império brasileiro, João Capistrano de Abreu exécuta ainsi son auteur, en qui il voyait un amateur besogneux, approximatif et excessivement prolixe. Pour Capistrano de Abreu, Pereira da Silva écrit de mauvais romans historiques, mais ne fait pas œuvre d'historien ${ }^{11}$. Il est loin de posséder le génie nécessaire pour élaborer un livre qui allierait l’imagination littéraire, l'écriture et la rigueur scientifique.

\footnotetext{
${ }^{9}$ Revista do Instituto Histórico e Geográfico Brasileiro, 1864, t.2, p. 400.

${ }^{10}$ SILVA, João Manuel Pereira da. Segundo Periodo do reinado de Dom Pedro I no Brasil. Narrativa histórica, Rio de Janeiro, B.L. Garnier, 1871.

${ }^{11}$ ABREU, João Capistrano de. "História da Fundação do Império Brasileiro », O Globo, 10 de março de 1877, in : Ensaios e Estudos, 4 a série, Rio de Janeiro-Brasília, Civilização brasileira/ INL, 1976, p. 37-41.
} 
Enfin, en 1880, un autre membre de l'IHGB, Prezalindo Lery Santos, auteur du Panteão fluminense ${ }^{12}$, une collection de biographies des natifs illustres de Rio de Janeiro, porta l'estocade à João Manuel Pereira da Silva qui, non seulement ne bénéficie pas des propos hagiographiques coutumiers dans ce type d'ouvrage, mais est taillé en pièces par son biographe. Pour le malheur de João Manuel Pereira da Silva, le livre de Prezalindo Lery Santos est recopié par la plupart des auteurs de dictionnaires biographiques, à commencer par Sacramento Blake.

Prezalindo Lery Santos reconnaît à Pereira da Silva d'immenses qualités d'orateur et rapporte que les conférences de celui-ci soulevaient l'enthousiasme. Il veut bien admettre son talent littéraire, cependant gâché par des fautes de grammaire. Pour le reste, c'est accablant. La carrière politique du Conseiller Pereira da Silva est brièvement relatée, mais ce dernier était dépourvu, selon son biographe, des vertus qui font l'homme d'Etat. Le pire concerne l'historien et mérite une longue citation: "Como historiador o Sr. Conselheiro Pereira da Silva tem um grande defeito, o maior de todos, a falta de critério com que escreve, aceitando como verdadeiros e cobrindo-os com a autoridade de seu prestígio literário fatos que não se acham comprovados, e muitos dos quais foram invenção das praças públicas em momentos de agitações políticas. Não pode o historiador aceitar levianamente as falsas opiniões criadas pelas oposições em seu plano de desmoralizar o objeto de seus ataques. (...)

Principalmente como historiador do primeiro reinado, não soube o Sr. Conselheiro Pereira da Silva guardar, ainda agora no fim de tantos anos, a imparcialidade e a frieza de historiador diante de acontecimentos que se passaram em uma época de efervescência política em que as paixões tudo cegavam. (...) E não somente em relação ao Sr. D. Pedro I, em várias ocasiões injustamente apreciado nesses trabalhos históricos, como em referência a muitos personagens que figuraram nos acontecimentos daquela época, deixou ele de proceder com a devida cautela. Ninguém ignora, por exemplo, as contestações que provocou a sua obra sobre o Segundo Reinado do Sr. D. Pedro I (sic).

São, pois, suas obras históricas eivadas todas de inexatidões e erros, que denotam a pouca atenção com que as escreve.”.

Prezalindo Lery Santos permet de comprendre le péché capital qui valut à João Manuel Pereira da Silva d'être chassé du paradis des historiens : avoir

\footnotetext{
${ }^{12}$ SANTOS, Prezalindo Lery. Pantheon fluminense. Esboços biográficos, Rio de Janeiro, Tip. G. Leuzinger \& Filhos, 1880.
} 
rapporté des faits et émis des jugements défavorables au premier empereur et son entourage pour la seconde partie de son règne, qui s'était terminé par la "Révolution d'avril" et l'abdication du souverain, ce qui rendait difficile un récit unanimiste.

João Manuel Pereira da Silva, bien que sympathique à D. Pedro $1^{\text {er }}$, distingue en effet deux moments dans la trajectoire brésilienne du monarque dans l'introduction du Segundo Período do reinado de Dom Pedro I no Brasil. Narrativa histórica. “Tratava-se na primeira época (1821-1825) de criar uma nova nação, fundar-lhe a independência e garantir-lhe a autonomia, obrigando Portugal, e os outros Estados soberanos da Europa e América, a aceita-la como igual e li$v \mathrm{v}^{13}$." et D. Pedro fut alors parfaitement à la hauteur des enjeux. En revanche, entre 1825 et 1831, "firmada a independência, concentrado o país em uma vida própria, espalhadas pelo povo as idéias e aspirações de liberdades públicas e privadas, de outros requisitos carecia o monarco, diversos dos que o haviam notabilitado durante o primeiro período do seu governo" ${ }^{\text {.14 }}$. L’historien évoque aussi, comme des faits ayant pesé sur la popularité de l'empereur, les scandales du règne, notamment les frasques notoires de l'empereur et de la marquise de Santos, et cite quelques libelles dirigés contre D. Pedro I comme celui-ci : "Vá Dionísio para Corintho, Vão os lusos para Lusitania e o Brasil será feliz”15. Nul doute qu'à un moment où l'inauguration par D. Pedro II de la statue équestre de son père au cœur de la capitale impériale était encore récente (1862) et signifiait la restauration symbolique de l'empereur déchu, le rappel des "erreurs politiques"- une expression employée par Pereira da Silva - du fondateur de l'Empire a dû fortement irriter au Palais et dans ses adjacences.

Varnhagen avait pu éprouver en son temps le “crayon fatidique” par lequel D. Pedro II annotait les projets et les ouvrages qui lui étaient soumis. Déjà, dans l'História Geral, l'empereur avait marqué sa désapprobation à propos de quelques expressions très légèrement critiques à l'égard de son père en les soulignant : D. Pedro I ${ }^{\mathrm{er}}$ “era franco e símpatico, mas nem sempre grave, e ás vezes caprichoso. Entusiasta pela glória, não conhecia ainda bem em que ela

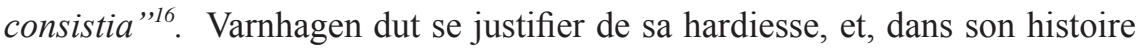

\footnotetext{
${ }^{13}$ SILVA, João Manuel Pereira da. Segundo Período do reinado de Dom Pedro I, op. cit., p. vi.

${ }^{14}$ Ibidem, p. vii.

${ }^{15}$ Cette citation date de 1823, est attribuée au parti des frères Andrada et est citée dans História da fundação do Império brasileiro 7... op. cit., p. 213.

${ }^{16}$ Lettre de Varnhagen à D. Pedro II, Madrid, 14 de julho de 1857, in : Correspondência ativa,
} 
de l'Indépendance, il visa principalement José Bonifácio de Andrada et ses frères qu'il détestait en partie pour des raisons familiales. C’était moins risqué que de s'en prendre au père de l'empereur régnant et ce, d'autant moins que les frères Andradas comptaient de très nombreux ennemis. De plus, lors de la parution tardive de cet inédit, sous la République déjà, l’histoire n’était plus du tout contemporaine et les acteurs avaient tous disparu. Comme censeur, Varnhagen n'eut droit qu'aux commentaires, en notes de bas de page, du baron do Rio Branco, lui-même décédé. Et João Manuel Pereira da Silva subit, seul, la malédiction de l'histoire de son temps.

\section{Deux histoires de I'Indépendance du Brésil}

En comparant la démarche de Pereira da Silva à celle de Thiers, Macaulay et Guizot, le premier secrétaire de l'IHGB était tombé juste. C'est grosso modo dans la perspective de l'Histoire du Consulat et de l'Empire d'Adolphe Thiers, dont la traduction paraît à Rio de Janeiro chez Garnier en 1865, que se situait notre auteur, fin connaisseur des milieux intellectuels et politiques français. Le dernier ouvrage de Pereira da Silva, Memórias de meu tempo (1895) - ses souvenirs politiques - semble faire écho aux Mémoires pour servir à l'histoire de mon temps, du ministre et historien français si apprécié des conservateurs brésiliens.

Les sept tomes qui constituent l'História da fundação do Império correspondent à un projet relativement bien organisé. Ils répondent à une intention narrative et explicative de ce que Pereira da Silva appelle la "révolution brésilienne"17 et s'accompagnent d'une réflexion sur la périodisation. Pour l'auteur, tout se joue en 1808 avec l'installation et le séjour de la Cour portugaise au Brésil. C'est avec l'ouverture des ports brésiliens au commerce des nations amies et l'installation de la famille royale à Rio de Janeiro que commence le processus de séparation qui s’achève avec la reconnaissance par le Portugal de l’indépendance du Brésil par le traité de 1825. De fait, cet événement spectaculaire jette les bases d'un nouvel État-nation en lieu et place du “continente brasílico”. Avant l'arrivée de D. João et de son gouvernement, "Nenhum laço prendia as capitanias. Não as ligava nenhum interesse. Estabelecida a sede do governo

coligida e anotada por Clado Ribeiro de Lessa, Rio de Janeiro, Ministério da Educação e Cultura, Instituto Nacional do Livro, 1961, p. 247.

${ }^{17}$ SILVA, João Manuel Pereira da. História da Fundação do Império Brasileiro, Rio de Janeiro, B.L. Garnier Editor, vol. 1, 1864, p.4. 
português no Rio de Janeiro, volveram as capitanias os seus olhos, a sua atenção, a sua obediência para a nova capital, situada no seio do seu próprio continente. Com a centralização política de todo o Brasil, com as relações sociais que daí nasceram, com as comunicações mercantis, que foram a sua consequencia, reuniram-se em um só Estado ; formaram uma nação homogenea de língua, de raça, de costumes e de interesses ; compuseram um império inteiro com vida própria, e perfeita emancipação da metrópole antiga ; e esqueram a sujeição que prestavam a Lisboa”18. Tout cela est le fruit de l'impact de l'inversion coloniale que relève João Manuel Pereira da Silva : "Converteu-se a colonia, por este feito, em metrópole dos estados da casa de Bragança”"19.

Le premier des sept volumes de la première édition n'est pour l'auteur qu'une longue “introduction” qui expose ses idées sur l'émancipation du Brésil et fait un tableau de l'histoire portugaise depuis la restauration de 1640 et du développement du Brésil au cours de la même période.

La méthode est rapidement évoquée : "Pesquisei, estudei, meditei, e comparei impressos e manuscritos, tradições orais e papéis do estado ${ }^{20}$ ». De fait, chaque volume de la collection est illustré par un très important cahier de documents, décrets, traités, mais aussi correspondance jusqu’alors inédite ${ }^{21}$. Quant aux "traditions orales” recueillies par Pereira da Silva, elles lui seront vivement reprochées par les plus hostiles de ses critiques qui n’y voient que de basses rumeurs. Ainsi, Prezalindo Lery Santos accuse l'historien de colporter les ragots : "Recolhendo os boatos das ruas e conventículos, coligindo as notícias adrede inventadas e preparadas pelos exaltados em seus planos revolucionários (...), o Sr. Conselheiro pus seu belo talento a serviço de uma causa má”22.

Une des grandes vertus de l'approche de João Manuel Pereira da Silva est l'intérêt qu'il porte à la circulation des hommes et des idées d'un continent ou d'un pays à l'autre, aux évolutions démographiques et économiques. Sa profession de foi liminaire dans une histoire nationale, par rapport à une historiographie royale, témoigne de l'influence qu'eut sur lui l'historiographie

\footnotetext{
${ }^{18}$ SILVA, João Manuel Pereira da. História da Fundação 2..., op. cit., p .45.

${ }^{19}$ SILVA, João Manuel Pereira da. História da Fundação 1 ..., op. cit., p. 129.

${ }^{20}$ SILVA, João Manuel Pereira da. História da fundação 1 ..., op.cit., p.4.

${ }^{21}$ Dans la seconde édition, en 3 volumes, le nombre de documents diminue drastiquement et ceux-ci se trouvent rassemblés à la toute fin de l'ouvrage.

${ }^{22}$ SANTOS, Prezalindo Lery. Pantheon fluminense. Esboços biográficos, Rio de Janeiro, Tip. G. Leuzinger \& Filhos, 1880, p. 488.
} 
romantique européenne “Tive sempre gosto pela historia. Não a quero, porém, para saber datas, estudar vidas de principes e personagens ilustres, e aprender o numéro das guerras e combates que se pelajarão. Prefiro que examina a fundo a sociedade inteira, que desce da cúpula elevada até o humildo chão do povo miúdo, discrimando as escalas, e camadas pelas quais se derrama a nação, e o sentir, o sofrer, o gozar e o aspirar de cada um dos súditos. Agrada-me mais a que desenha os traços da administração pública, no mais largo sentido desta palavra, social, politica, civil e economica. Assim compreende a historia o povo e a nação toda, e a apresenta de perfil, de face, no corpo, na alma e no espírito"²3.

Contrairement à cette déclaration d'intention, le "povo miúdo" apparaît bien peu, dans l'œuvre de João Manuel Pereira da Silva, ou alors sous forme de masses exaltées, livrées à l'anarchie. Quant aux esclaves, ils sont selon lui un mal nécessaire pour l'économie nationale et, conformément aux poncifs habituels, sont mieux traités qu'ailleurs ${ }^{24}$. Les “sauvages” cesseront de l'être quand ils se dilueront dans une immigration européenne massive que Pereira da Silva appelle de ses vœux. Sur ces points, João Manuel Pereira da Silva se montre banalement fidèle aux représentations dominantes de la société brésilienne sous D. Pedro II.

Les "peuples", comme dépositaire de l'âme nationale, y figurent tout de même et savent même remplacer les rois défaillants. C’est de cette manière que l'historien rend compte de la crise de la monarchie espagnole, de l'insurrection contre l'invasion française et de l'ingratitude du piètre et borné Ferdinand VII.

Car, pour Pereira da Silva, l'indépendance du Brésil n'est pas dissociable des turbulences européennes et américaines du tournant des XVIII ${ }^{\mathrm{e}}$ siècle et $\mathrm{XIX}^{\mathrm{e}}$ siècle. Des développements nourris sont consacrés aux affaires du Rio de la Plata, si mêlées à celle de la monarchie portugaise et offrent l'occasion de comparaisons intéressantes, tant avec le Brésil qu’avec les États-Unis. Contrairement au vice-royaume de La Plata, ces derniers ont connu une séparation d'avec la métropole, mais certainement pas de "révolution”. Il existait dans les treize colonies britanniques, comme en Angleterre, des libertés et des institutions représentatives qu'il s'est agi d'adapter simplement. Les Espagnols, dans

\footnotetext{
${ }^{23}$ SILVA, João Manuel Pereira da. História da Fundação 1..., op. cit, p.7.

${ }^{24}$ En 1845, dans un recueil qui rassemblait des articles publiés dans divers journaux, Pereira da Silva déclinait tout l'argumentaire destiné à préserver la traite négrière et l'esclavage, João Manuel Pereira da Silva, Inglaterra e o Brasil.. Trafego de escravos por um deputado, Rio de Janeiro, Tip. do Brasil, 1845.
} 
la péninsule ibérique comme aux Indes, n’étaient que les “esclaves obéissants” d'un gouvernement inquisitorial, les sujets soumis d'une monarchie absolue, rétrograde et superstitieuse. En se libérant de Madrid, les nouvelles républiques ont dû tout imaginer et inventer une législation, des us et coutumes, des systèmes politiques... Cette table rase est sans doute l'origine des guerres civiles et des désordres qui ont ravagé la région pendant la première moitié du XIX ${ }^{\mathrm{e}}$ siècle ${ }^{25}$.

João Manuel Pereira da Silva accorde une place tout à fait détaillée au versant portugais de l'indépendance, ce qui le distingue également d'autres auteurs brésiliens ayant abordé la période. Il relate minutieusement les invasions françaises successives, les souffrances liées à la guerre, la fin de l'Exclusif et le traité de 1810 avec la Grande-Bretagne qui semblent signifier la ruine du commerce péninsulaire, l’humiliation éprouvée par le royaume d’être rabaissé au rang de colonie, l'impopularité du conseil de Régence et de la protection britannique, et le ressentiment éprouvé à l'encontre du Brésil qui fait figure de profiteur des malheurs du temps.

Les analyses sont soutenues par une vision politique cohérente qui reflète le "juste milieu” auquel aspire une bonne partie des élites impériales et du parti conservateur qui répugne tant à l'absolutisme qu'à l'anarchie. Pereira da Silva estime que la monarchie préserva l'unité territoriale héritée de l'Amérique portugaise et façonnée par la présence de la Cour de 1808 à 1821. Qu’une bonne constitution et un gouvernement représentatif fondé sur la compétence et le mérite garantissent les libertés et éloignent les trois formes de despotisme identifiées par notre auteur : le royal, l'aristocratique et le démocratique ${ }^{26}$. Que l'éducation et la vigueur de la création littéraire et artistique sont une des conditions du progrès.

L'histoire de l'indépendance de Varnhagen est en comparaison beaucoup moins aboutie et manque singulièrement de souffle. Elle n'est qu'un appendice de l'História Geral dont la 2e édition de 1876 abordait déjà la période 1808-1820. Selon le découpage du vicomte de Porto Seguro, le processus de séparation entre le Brésil et le Portugal est donc sectionné et l'attention se concentre surtout sur les événements de 1821 et 1822.

Varnhagen est plus disert que Pereira da Silva sur ses sources, fidèle à sa réputation de chercheur de documents. La force de son livre tient selon lui à la

\footnotetext{
${ }^{25}$ SILVA, João Manuel Pereira da. História da Fundação 4..., op. cit., p .269.

${ }^{26}$ SILVA, João Manuel Pereira da. História da Fundação 7 ..., op. cit, p.111.
} 
"pureza das fontes e abundância de documentos que se tiveram presentes, além dos publicados por Cairú, e aproveitados por Pereira da Silva, a saber :

01- as coleções, mais ou menos completas, dos períodicos do tempo, e com especialidade o Reverbero, a Malagueta, o Espelho e o Regenerador”

02 - todas as publicações avulsas, não periodicas, do mesmo tempo, que são muitíssimas, e hoje raras de encontrar.

03- Várias informações verbais, recolhidas desde 1840 pelo autor (e desde logo por ele protocolizadas), em conversações com vários corypheus da independência que conheceu e tratou, incluindo os marqueses de Paranaguá, Valença, Resende, Monte-Alegre et Sapucaí, e também Januário, Ledo, Vergueiro, Rafael Tobias e outros.

04 - Finalmente, as importantissimas correspondências oficiais dos agentes diplomaticos e consulares espanhol, francês, inglês e austriáco, desde 1821 à 1825 , consultadas pelo autor”27.

Varnhagen ne répugne pas à faire l'histoire de son temps à l'aide de témoignages, mais cite ses informateurs, contrairement à Pereira da Silva. Il apporte aussi des éléments d'érudition qui font sans doute défaut sur certains points à son rival explicitement mentionné dans l'introduction, mais il renonça, pour des raisons obscures, à peaufiner et publier son ouvrage. Était-il trop occupé à prouver l'origine troyenne des Tupis du Brésil, quête qui occupe ses dernières années? ${ }^{28}$ Craignait-il de toucher à l'histoire moderne et à ses contestations sans fin? Jugeait-il finalement son travail inférieur à celui de Pereira da Silva, malgré la réputation de manque de rigueur de ce dernier et la réputation inverse dont lui-même jouissait?

L’histoire de l'indépendance n'était pas, de toute façon, le moment privilégié par le régime impérial et ses historiens dans la construction nationale. Ces derniers se penchèrent de préférence au-dessus du berceau de la nation, distincte, dans la plupart des esprits, de la fondation de l’Empire. Le Brésil, comme nation dotée d’un génie et non comme un État-nation fondé sur un pacte politique,

\footnotetext{
${ }^{27}$ RIHGB t. 79 1916, p.6.

${ }^{28}$ VARNHAGEN, Francisco Adolfo de. L'origine touranienne des américains tupis-caribes et des anciens égyptiens indiquée principalement par la philologie comparée: traces d'une ancienne migration en Amérique, invasion du Brésil par les Tupis, etc... Vienne, s.e. 1876.
} 
était né et avait reçu le baptême en avril 1500 sur les plages de Bahia. Il est tout à fait significatif que Varnhagen, "primeiro historiador da nação brasileira (na ordem cronólogica e no merecimento)”29 ait été créé par D. Pedro II, baron, puis vicomte, de Porto Seguro et que la Première Messe, décrite par Pero Vaz de Caminha et peinte par Vítor Meireles, ait connu une telle ferveur.

L'histoire récente exposait bien davantage celui qui s'y risquait à une levée de boucliers. L'examen des circonstances dans lesquelles avait été fondé l'Empire, pour reprendre le titre de Pereira da Silva, mettait à nu les contradictions du régime et rappelait désagréablement les luttes politiques qui avaient émaillé la première moitié du XIX ${ }^{\mathrm{e}}$ siècle brésilien. La “Révolution d'avril” 1831 avait fait rejouer les failles de l'Indépendance. Sous le règne personnel de son fils, avec la restauration du pouvoir modérateur, la figure de D. Pedro $1^{\mathrm{er}}$ était devenue sacrée tout en demeurant hautement polémique. Les mésaventures de João Manuel Pereira da Silva reflètent en définitive les difficultés de l'institutionnalisation de l'histoire contemporaine, toujours soupçonnée d’illégitimité et d'esprit partisan. Elles traduisaient aussi les limites de l'espace critique sous D. Pedro II, espace critique sans lequel il ne saurait y avoir d'histoire contemporaine.

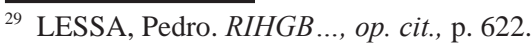

